\title{
Seismicity Management at Hill 50 Gold Mine, Western Australia
}

\author{
F.R.P. Basson AMC Consultants Pty Ltd, Australia
}

S. Van Der Merwe Mt Magnet Operations, Harmony Gold (Australia) Pty Ltd

\begin{abstract}
Analysing seismic information is a time-consuming task requiring substantial engineering effort. Computer codes are available to speed up the analysis and help relate seismicity with predetermined variables. The long-term goal would be to predict seismic events with sufficient confidence for input in short-term mine design, this remains however elusive.

This paper presents a case study on the deepest Western Australian gold mine (Hill 50), where multiple methods were applied to unravel the seemingly random nature of periods of high seismic activity in the mine. Numerical modelling results were compared with outcomes and conclusions drawn on the applicability of different analysis methodologies.
\end{abstract}

\section{Introduction to Hill 50}

Hill 50 Gold Mine is located some 550 kilometres north-east of Perth in the Murchison region of Western Australia. In its history, the mine has poured some 2 million ounces of gold and has reached a current depth of 1510 metres below surface.

Seismicity has become part of the routine management systems utilised during daily mining activities. It is not uncommon for the operation to experience local magnitudes of up to 1.9 on the ISSI scale after stope blasting activities have been carried out. Whilst most of the seismicity is located in the rock mass surrounding the preceding stope blast, it is still common for seismicity to be encountered on levels within the mine situated up to 500 metres shallower. It is for this reason that a large amount of time has been spent analysing the Hill 50 seismic dataset so as to try and establish which geological structures within the mine pose particular risk should they be mobilised.

\section{Seismicity background}

In the authors' time at Hill 50 Gold Mine, the only mining method employed for orebody extraction was that of uphole benching with 25 metre lifts. Stope voids were post filled with unconsolidated waste fill purely as a waste transport reduction method and as such provided very little support to the surrounding rock mass.

Literature published indicates that the onset of micro-seismicity at the mine was experienced at a depth of some 1000 metres below surface. This has continued to increase as mining has progressed deeper, with current depths experiencing seismic events closer to excavation perimeters. The understanding of mechanisms driving seismicity at the mine has changed over time and this has come as a result of altered mining layouts and pillar designs.

Historically, pillar layouts were considered for no other reason than to prevent unconsolidated waste fill from entering producing stopes. As extraction of the orebody increased so did mining induced stress levels to the point that failure of the pillars was occurring in a seismic manner. The first large assessment of seismic occurrences at Hill 50 focussed on pillar failures throughout the mine and aimed to establish suitable pillar failure criteria (Sharrock, 2005). However, in February 2006, a large, erratic and widely dispersed seismic response was experienced with large seismic events occurring up to 500 metres higher up in the mine from current production levels. It was then that research was conducted into highlighting geological structures within the mine, which are prone to the erratic clustering of seismicity. Contouring of Peak Particle Velocities (PPV) was conducted in an attempt to highlight areas where seismicity levels were high relative to the extent of stoping. The high levels of seismicity might therefore have indicated the presence of seismically active structures. 


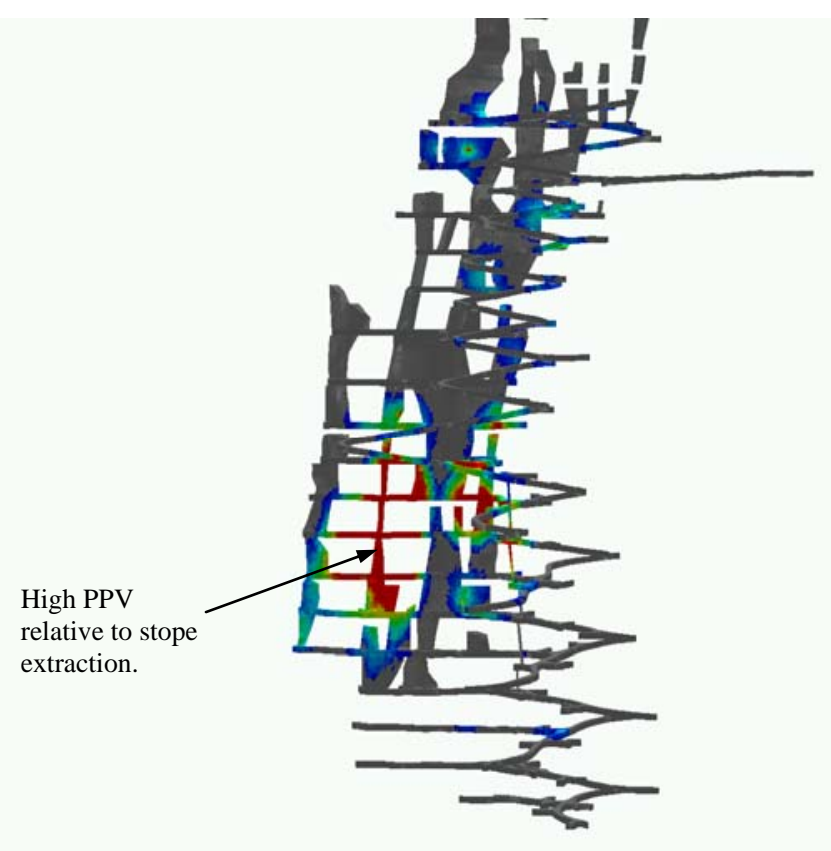

Figure 1 PPV contouring with a threshold value of $45 \mathrm{~mm} / \mathrm{s}$ (after Basson, 2006)

Following the above mentioned research, various engineering controls were implemented so as to facilitate improved management of seismicity at the mine. A system of non-yielding crown and rib pillars was designed to reduce shear stress occurring along geological structures prone to seismicity (Albrecht, 2006). Furthermore, the installation of dynamic type ground support and the use of exclusion periods in nondynamically supported ore drives, following stope blasts was implemented (Van der Merwe, 2007). Although successful to a large degree, there is still the unexplained erratic nature of seismicity at Hill 50, sometimes up to five days after a stope blast has been conducted. It is therefore the purpose of this research to better understand how stress change within the mine might be driving seismicity higher up.

\section{Background for the analysis}

The Australian Centre for Geomechanics (“ACG”) requested the authors to engage in a research project. The aim of the project was to add value to seismic event interpretation from numerical modelling.

Tasks included calibration of the far-field stress orientation from seismic event locations, calculation of principal stresses at the event locations, and to resolve shear and normal stresses for geological structures at these locations. The authors addressed the tasks and included calculation of excess shear stress ("ESS") on structures and looked into the ability of neural networks to correlate relevant input data with results gained during the study.

\subsection{Identification of seismically active structures}

The Hill 50 geology is rounded and planar structures are seemingly not present. Known structure planes at Hill 50 also do not align well with the recorded seismic events.

Kijko et al. (1999) developed software called RMD that runs within the MatLab environment to identify potential structures from the seismic information as part of a SIMRAC-project. The method proposed consists of moving the hypocentre of events within their confidence ellipsoids to obtain a simplified pattern of the seismic event cluster. RMD only retains repositioned events within the confidence ellipsoids and ignores isolated events. The resultant simplified pattern might then be interpreted for a rock mass discontinuity. A full description of the approach can be downloaded from the SIMRAC-web page.

The authors filtered the seismic information and rejected all suspicious events that could contaminate the database. Only events recorded by four or more sensors and with a location error of less than $10 \mathrm{~m}$ were retained with further filtering done on selected event parameters. 
RMD has different options to calculate the location error and relocation of events. The preferred choice is to specify sensor locations and a standard deviation (SD) of the P-wave arrival time, mean P-wave velocity and SD of the P-wave velocity. The second alternative is to specify an average error for event locations.

The authors opted for the second approach and assumed a fixed error of $10 \mathrm{~m}$ as only events with a calculated location error of less than $10 \mathrm{~m}$ were selected from the database. The relocation amount is a function of the error specified and the number of relocation iterations. For this study, the authors selected a single iteration.

Results from RMD are shown in Figure 1, with the transparent events marking the recorded event positions, and the solid events the relocated event positions.

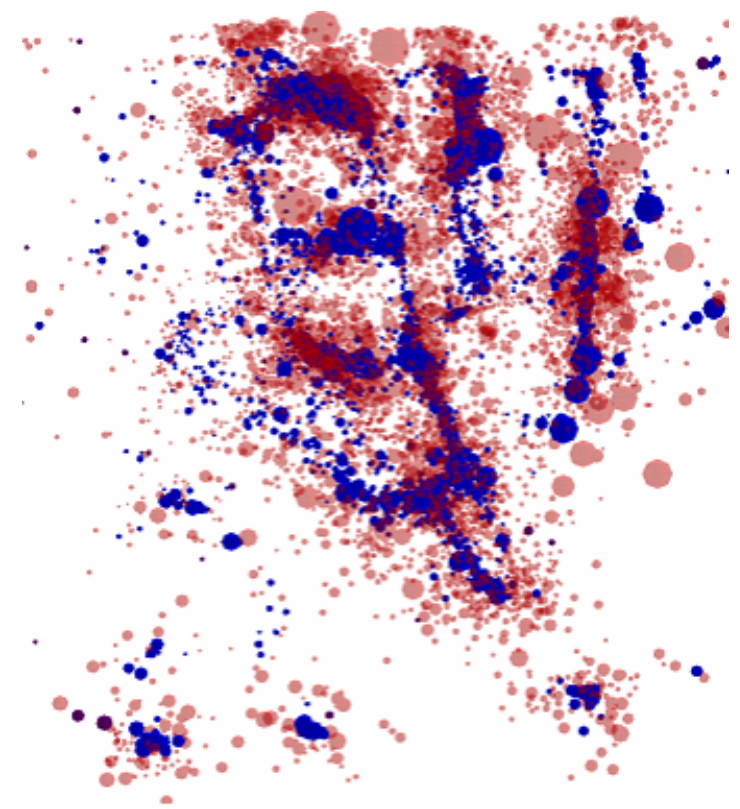

Figure 2 Recorded and relocated events from RMD

Events were split into four groups and planes deduced from the RMD results for each time period. Three potential geological discontinuities were identified in multiple data sets and found to be in good spatial agreement (see Figure 2). The planes conformed well to the stoping volumes and were extended to cover a suitable area for numerical modelling purposes.

The planes deduced from the RMD-results are referred to as Structure 1, Structure 2 and Structure 3 as showed in Figure 3. 


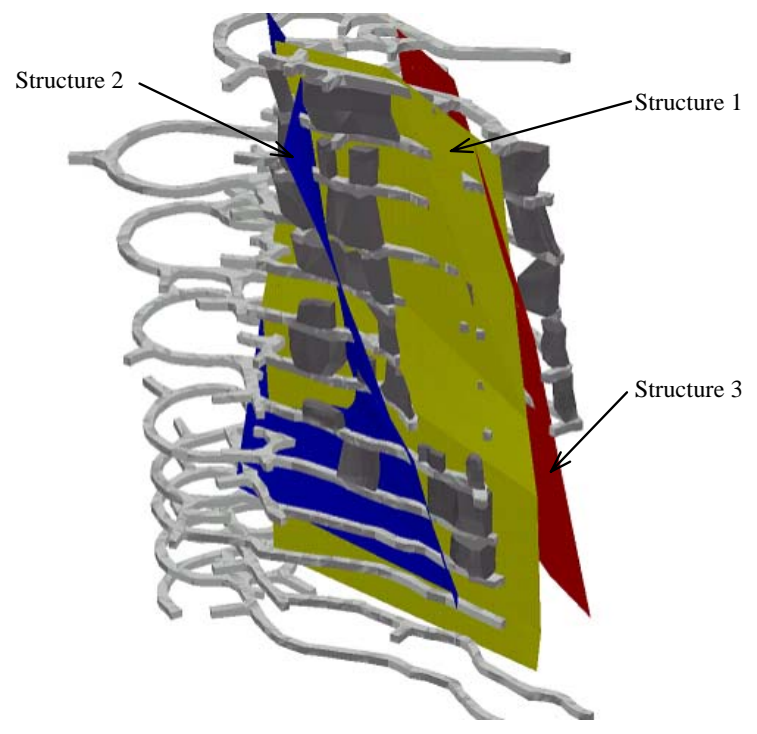

Figure 3 Planes deduced from the RMD results

\subsection{Map3D Model}

A Map3D model taking account of all blasts (except one) from 1 January 2006 to 1 April 2007 was constructed. The dismissed blast was the smallest recorded blast $\left(70 \mathrm{~m}^{3}\right)$, which was removed to accommodate the Map3D limit of 40 mining steps.

\subsection{Seismic Response to Mining}

Figure 4 shows the correlation between extraction and the radiated energy from the seismicity over the period studied. There is an overall correlation with an increase in radiated energy as stoping progresses. The correlation is less obvious in smaller time frames, with the biggest difference occurring around the start of 2007 at Mining Step 31.

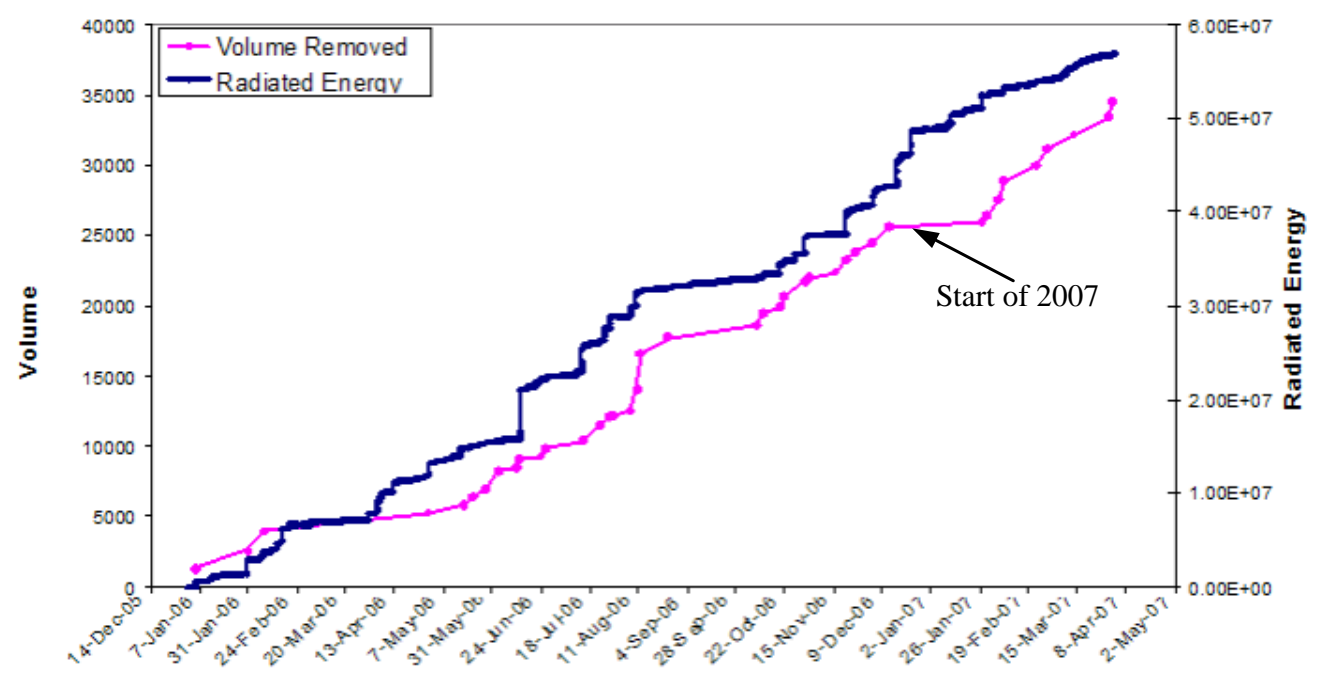

Figure 4 Seismic response to mining 
Some mining steps triggered a regional seismic response that far exceeded the typical responses for other extraction steps. Figure 5 shows the events recorded for Mining Step 31 where $1115 \mathrm{~m}^{3}$ of rock was blasted with two rings.

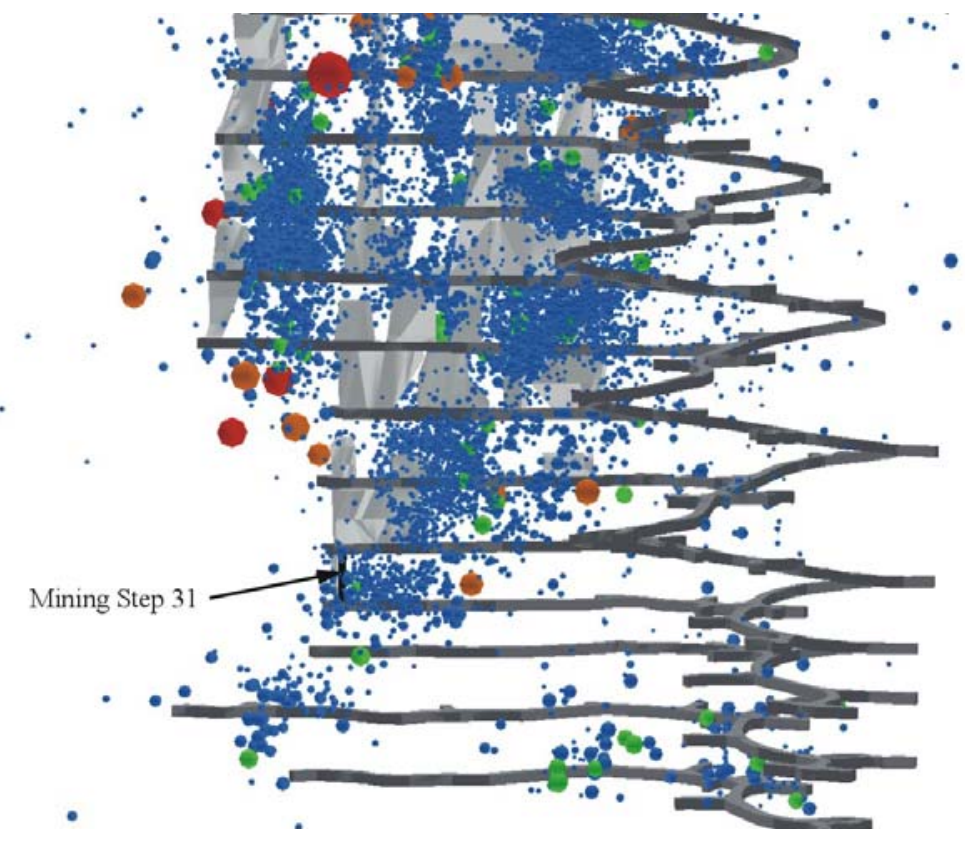

Figure 5 Seismic response to mining step 31

\section{$4 \quad$ Analysis undertaken}

\subsection{Far field stress calibration}

The stress field normally used by Hill 50 for numerical modelling was deduced from multiple stress measurements over years. The latest measurement was done in December 2006 and agrees with the expectation from previous measurements. This stress state was slightly adjusted for the far-field stress calibration analysis. Sigma 1 was assumed to be horizontal and Sigma 3 vertically down. The azimuth of the stress tensor was rotated in increments of $20^{\circ}$ from $295^{\circ}$ to $135^{\circ}$ as shown in Figure 6.

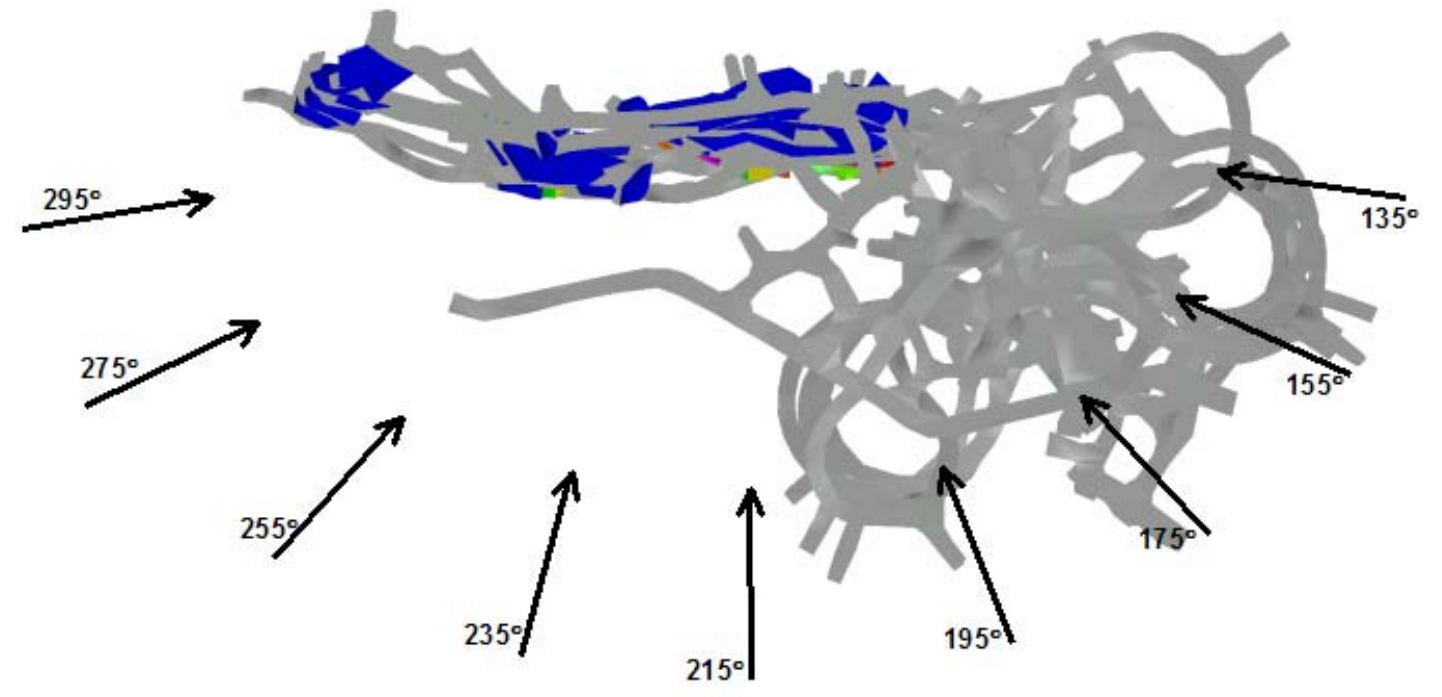

Figure 6 Stress directions tested 
When seismic locations are accurate, they could potentially be used to determine the far field stress orientation by multiple stress analysis on the same geometry. The best match between contours of maximum shears stress $1 / 2\left(\sigma_{1}-\sigma_{3}\right)$ and observed seismicity should point out the far field stress.

The authors made use of the seismic events recorded for mining step 31 and correlated the maximum shear stress with recorded events on two planes. The upper plane was between levels 19A and 19 (away from the active stope) and the lower plane between levels 20 and 21A (on the active stoping face horizon). The seismic events that occurred between levels 19A and 19 were projected to the Map3D grid plane in the centre of these levels, and the same was done for the events between levels 20 and 21A.

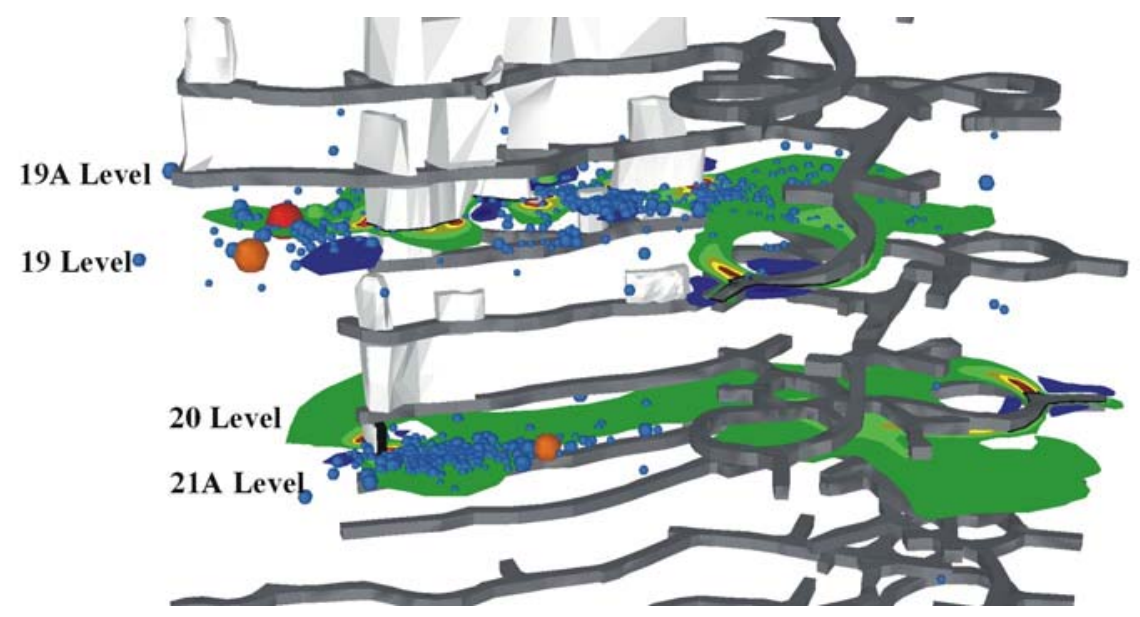

Figure 7 Maximum shear stress and recorded seismic events

The events between 19A and 19 Levels were found to have no correlation with the maximum shear stress contours. A better visual correlation was found around the active stoping faces for some of the stress orientations. The strongest and weakest correlations were found at Sigma 1 azimuths that differ by around $100^{\circ}$. As only $20^{\circ}$ increments were tested, the difference in azimuth was close to the expected $90^{\circ}$.

The strongest correlation agrees with the stress state orientation obtained from HI-cell measurements.

\subsection{Rock mass failure criterion}

Wiles et al. (2001) calculated stresses at seismic event locations to determine rock mass failure criteria. This was done at the AECL underground research laboratory in a controlled environment and a dense seismic array to record small magnitude events close to the excavation boundary. They found that these stresses fit a line where the difference between the major $\left(\sigma_{1}\right)$ and minor $\left(\sigma_{3}\right)$ principal stress is a constant value:

$$
\sigma_{1}-\sigma_{3}=\text { constant }
$$

For this investigation, the stresses were determined for seismic events recorded within a $50 \mathrm{~m}$ radius cylinder and a $50 \mathrm{~m}$ elevation difference from the blasted stope centres for all 40 mining steps. The authors considered that these events could potentially have been triggered because of stress redistribution within the rock mass. Events further away were assumed to be from stress redistributions on structures not captured by the stress analysis.

Most of the seismic events occurred between Sigma 1-values of 60-80 MPa and Sigma 3-values of 20-30 MPa. Few events were recorded with low Sigma 3 values and might indicate that clamping stress is required for favourable seismic conditions.

The seismic event magnitudes are represented with size and colour in the Sigma1 versus Sigma 3 plot shown in Figure 7. The stress states for the bigger events do not differ from those of the smaller events. The events with local magnitudes exceeding 1 form a line represented by $\sigma_{1}-\sigma_{3}=42 \mathrm{MPa}$ within the block of values where seismicity commonly occurs. The number of incidents is too few to draw conclusions from this observation. 


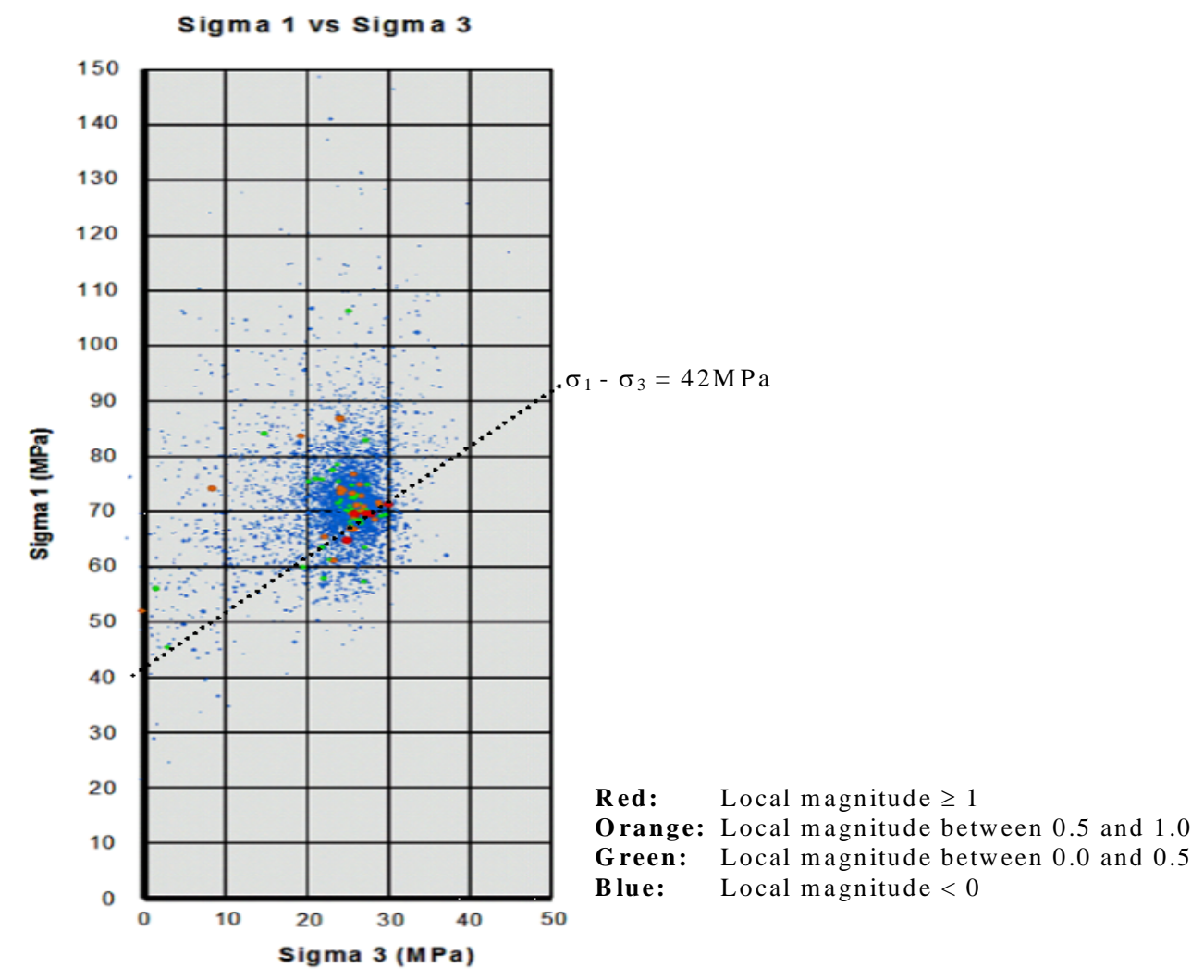

Figure 8 Maximum shear stress and recorded seismic events

\subsection{Normal and shear stresses on planes}

The stress tensor was determined at all event locations within the previously mentioned $50 \mathrm{~m}$ cylinders around active stoping faces for all mining steps. The shear and normal stresses at the event locations were resolved for plane orientations ranging all dip and dip-directions values, and the correlation for each dataset calculated with the objective to identify patterns in the values. An example result is presented in Figure 9.

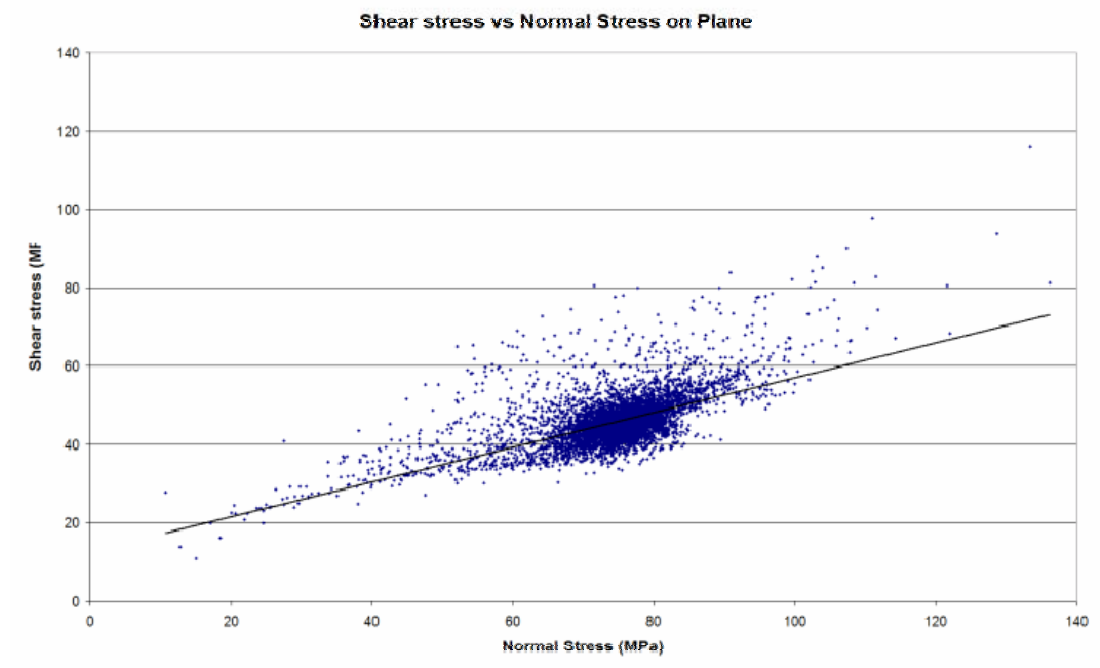

Figure 9 A dip and dip direction plane with a good correlation in the data set

The least square correlation values correlate well with visual correlation in the dataset. Some trend lines have no physical meaning from a Mohr-Coulomb failure criteria point of view. For example, a trend line with a negative slope will require a decreasing friction angle with an increase in normal stress. High 
correlation values suggest planes where the stress values line up with little scatter and a potential pattern could exist in the dataset. Planes parallel to the major principal stress and perpendicular to the minor principal stress directions (sub-horizontal) show little difference with changing dip direction (along line 'AA' in Figure 10). Preferential orientations exist for good correlation in the data and the highest values calculated at $40^{\circ} / 140^{\circ}$ and $50^{\circ} / 140^{\circ}$.

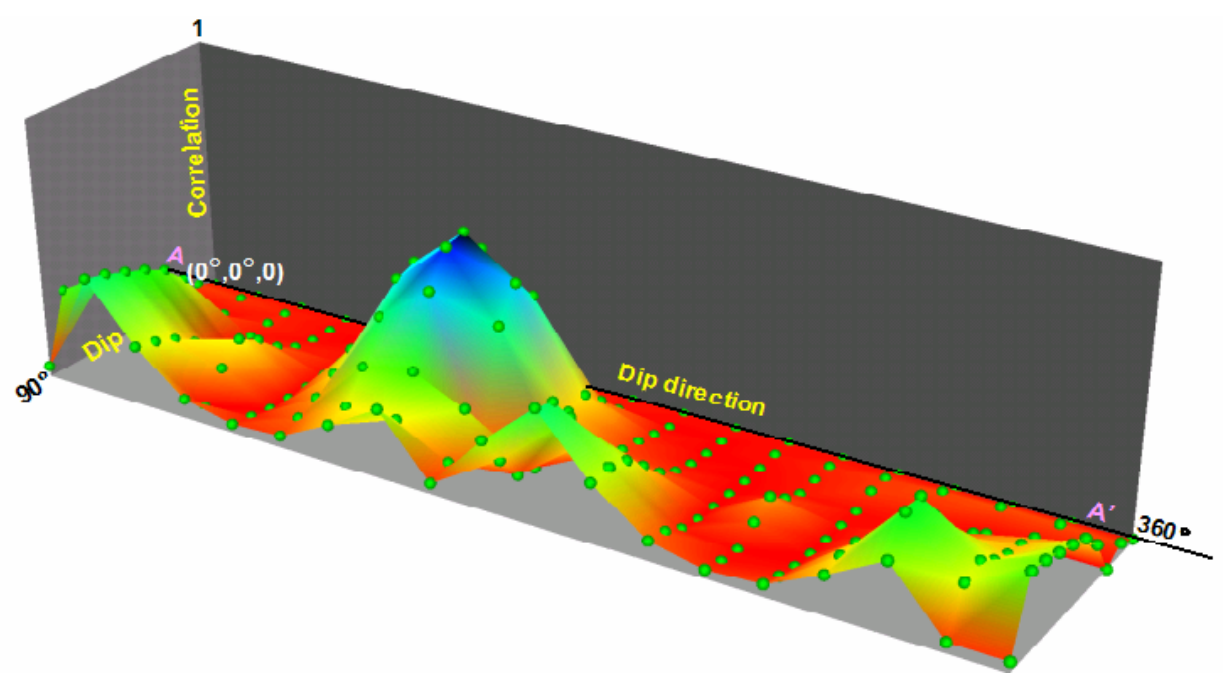

Figure 10 Data correlation for different potential planes

Normal and shear stress values were tested for average dip and dip-direction values of the identified planes in Figure 3. The data does not align well on all planes. Structure 2 has the best correlation and the alignment suggests a failure criterion with an average friction angle of $10^{\circ}$ and a cohesion of $50 \mathrm{MPa}$. The cohesion value is considered too high for a pre-existing fault plane, except when failure of asperities controls the seismic emissions.

\subsection{Excess shear stress calculation on planes}

ESS-values were calculated on all failure planes to compare with event occurrences. Resultant stresses for the grid planes were exported from Map3D as text files to enable manual manipulation of the data.

ESS is defined as the difference between the prevailing stress before slip and the dynamic strength of the plane. Ryder (1988) considers that values for cohesion could be between $0 \mathrm{MPa}$ and $10 \mathrm{MPa}$ and friction angles between $30^{\circ}$ and $32^{\circ}$ for in situ static strengths. Under dynamic conditions, cohesion is assumed to be negligible and the dynamic friction angle estimated at roughly $30^{\circ}$ by Ryder.

Some of the increased seismic activity time periods can be correlated to increased shear stresses generated on planes. Other high activity periods could not be related to the modelled results. One possible explanation would be that asperities prevented ride on the planes until their strength was exceeded. Two factors could contribute towards the failure of these asperities, increasing shear stresses and a time-dependant weakening of the asperities under load. A single asperity failure could then result in "domino" effect, where redistributed stresses are transferred to other asperities and result in a chain of failures.

The comparison was repeated for events recorded within $50 \mathrm{~m}$ from the active stope to determine if a better correlation could be found for events closer to the active area. The correlation for these events was as weak as for the regional events. 


\section{Correlation between input and output data}

\subsection{Neural networks}

Columns of input and output data were imported into neural network software to evaluate the ability of this technology to relate combinations of input to the measured seismic response (output). The software used is called EasyNN-Plus and has the ability to grow multi-layer neural networks from data in a grid. The neural network input and output layers are created to match the grid input and output columns. Hidden layers connecting the input and output layers are automatically grown to obtain the optimum number of nodes.

The data can be queried for predictive outcomes after network training, but was not used in that way for this investigation. Two results from neural network analysis are the "importance" and "sensitivity" of the input data. Importance shows the relative weighting of each input on the output data. Sensitivity shows how much the output changes with variability in each input value. Importance was used to discover the most prominent factors in producing the seismic outcomes recorded, and the sensitivity to evaluate the results against human judgement.

The process was repeated for regional and local seismic responses. The input data was the relative position of the blasted stope (defined by a number), the volume extracted, the ESS-values for the individual planes and combined ESS-values. The output data was the maximum recorded local magnitude, number of events greater than local magnitude 1.0 and 0.5 (two columns), the cumulative moment and radiated energy.

\subsubsection{Regional response}

The neural network associated absence of ESS on Structure 3 with the potential for increased seismicity. This reverse correlation is not sensible and the ESS-values for Structure 3 removed from the input for following runs. Visual inspection of the data indicated that regional seismicity could sometimes be related to the "O1 South" stopes, but the neural network could not find a similar correlation.

The authors concluded that the neural network approach is not reliable to identify the relative importance of input parameters to the regional seismic response.

\subsubsection{Local response}

The neural network results signalled that the most important input is the volume extracted, with the modelled ESS on Planes 3 and 1 also of high importance. A concern in the results is the importance given to the incremental ESS on Plane 3 for the same reason as mentioned for the regional response.

After omitting Plane 3, the volume extracted is still signalled as the most important input parameter. This is a more favourable result than for the regional case, indicating that the volume extracted could be successfully related on both occasions to the output data. The relative stope position gave the weakest correlation in both cases and is thus not identified by the software as important to quantify the local response.

It appears as if the volume extracted is better related to the seismic response around the active stopes than the calculated ESS-values on the structures identified with RMD.

\section{Conclusions}

The following were found during the study:

- A general correlation between stoping volumes extracted and radiated seismic energy. The correlation is strong over long time periods (months or years), but less obvious in smaller time frames (days or weeks).

- The radiated seismic energy is sometimes because of a large number of small events and in other instances due to fewer but higher magnitude events.

- When the different virgin stress states are applied to the stoping, the seismic events close to an active stope correlate reasonably well with the maximum shear stress values around the stope. The stress state of the strongest correlation also agrees with the stress state from HI-cell stress measurements. 
- The stress state of most seismic events occurred between Sigma 1-values of 60 to $80 \mathrm{MPa}$ and Sigma 3 values of $20 \mathrm{MPa}$ to $30 \mathrm{MPa}$. The stress states for the bigger events do not differ from those of the smaller events.

- Plots of shear and normal stress values on potential planes through the events line up better for some planes than others. The shear and normal stress values from planes deduced from RMD did not line up well.

- Few of the increased seismic activity time periods could be related to increased shear stresses on planes.

- Visual inspection of the seismic data suggests that stopes to the south are potentially more inclined to produce a regional response than stopes to the north.

- Inspection reveals that extraction volumes correlated reasonably well with the generation of large seismic events on a regional and local scale.

- Neural network analysis software gave consistent results for the local seismic response to stoping operations, but failed to give consistent results for the regional seismic response.

\section{Acknowledgements}

Harmony Gold Management, for their support in facilitating the research project. Also to the ACG and AMC Consultants for the time spent analysing data in an attempt to increase knowledge about seismicity management within Australia.

\section{References}

Albrecht, J.A. (2006) Hill 50 Site Report, AMC Consultants internal report to Harmony Gold, May 2006.

Basson, F.R.P. (2006) Seismic Occurrences at Hill 50 and implication for future workings. AMC Consultants internal report to Harmony Gold, April 2006.

EasyNN-plus documentation (2004) Web site: http://www.easynn.com.

Kijko, A., Lasoki, S. and Retief, S.J.P. (1999) Identification of Rock Mass Discontinuities in a Cluster of Seismic Event Hypocentres. SIMRAC-report GAP 622. Download from http://www.simrac.co.za/report/Reports/thrust3/gap622/gap622.htm.

Ryder, J.A. (1988) Excess Shear Stress in the Assessment of Geologically Hazardous Situations. Journal of the South African Institute of Mining and Metallurgy, Vol. 88, No. 1, pp. 27-39. January 1988.

Sharrock, G. (2005) Seismicity and the Stability of Pillars at Hill 50 Gold Mine. AMC Consultants internal report to Harmony Gold, February 2005.

Van der Merwe, S. (2007) Hill 50 Mine Ground Support Strategy Review. Internal Harmony Gold report, May 2007.

Wiles, T.D., Lachenicht, R. and Van Aswegen, G. (2001) Integration of Deterministic Modelling with Seismic Monitoring for the Assessment of the Rockmass Response to Mining: Part 1 Theory. $5^{\text {th }}$ Symposium on Rockbursts and Seismicity in Mines (RaSiM 5), South Africa. September 2001. 